\title{
Article \\ A Lagrangian tool for simulating the transport of chemical pollutants in the Arabian/Persian Gulf
}

\author{
Raúl Periáñez (D0000-0003-2586-3884
}

Dpt. Física aplicada I, ETSIA, Universidad de Sevilla, Spain; rperianez@us.es

\begin{abstract}
A rapid-response Lagrangian model for simulating the transport of a chemical pollutant in the Arabian/Persian Guls is described. The model is well suited to provide a fast response after an emergency due to an accident or a deliberate spill. Baroclinic circulation was obtained from HYCOM ocean model and tides were calculated using a barotropic model. The interactions of pollutants with sediments (uptake/release processes) were described using a dynamic approach based on kinetic transfer coefficients and a stochastic numerical method. Some examples of model applications are shown.
\end{abstract}

Keywords: Arabian/Persian Gulf; Lagrangian model; pollutant transport; tide; baroclinic circulation

\section{Introduction}

The Arabian (or Persian) Gulf -APG from now on- (Figure 1) is exposed to a number of pollutant threads. This constitutes a significant concern for countries surrounding the APG since desalination plants are the main freshwater source to them. In addition, commercial and subsistence fisheries provide a living for a large sector of the coastal population [1].

Some of the sources of pollution [2] include desalination plants (which are a source of radium in the brine discharged to the sea) and phosphate industry (in phosphogypsum waste). Oil spills are relatively common in the APG, in addition to the massive releases during the 1991 Gulf War. In addition to this, the APG, Strait of Hormuz and Gulf of Oman are one of the most important waterways in the world, thus exposed to pollution incidents due to shipping activities. Recently, there has also been concern about the nuclear power plants (NPP) which are operating along the APG coasts. There are two operational NPPs in the region, Bushehr in Iran and Barakah in UAE, whose unit 1 was connected to the power grid in summer 2020. About seventeen more are planned in the Kingdom of Saudi Arabia, with the intention that they are operational by 2030 [3].

Consequently, it is relevant to have a numerical model able to assess the effects of pollutant releases (both chemical and radioactive) into the APG. Although some models are described in literature concerning the dispersion of oil spills in the APG [4], this is not the case for chemical pollutants. The objective of the present model consists of filling such gap.

APERTRACK is a Lagrangian three-dimensional model which simulates the transport of pollutants in the APG. It can be applied to any chemical pollutant since a decay process is included (which can be due to radioactive decay or another decay processes, like biodegradation). Furthermore, interactions of pollutants with sediments are included in a dynamic way, by means of kinetic transfer coefficients. It is known that heavy metals and some radioactive elements $(\mathrm{Pu}, \mathrm{Th}$, etc) present a high affinity to be fixed to sediments, thus these processes are relevant for this kind of contaminants.

The model code is written in FORTRAN, all required input data is open and open source software is used for result visualization. In particular, provided scripts to draw contaminant concentration maps have been developed for GNU Octave, version 5.2.0, free software. Octave is available for Windows and Linux computers. The model with all required input data and scrips for visualization may be free downloaded from the author webpage www.personal.us.es/rperianez. Main characteristics are presented in Table 1.

A brief description of the model, including required input data and model output, is given in section 2. Next, some application examples are provided in section 3. 


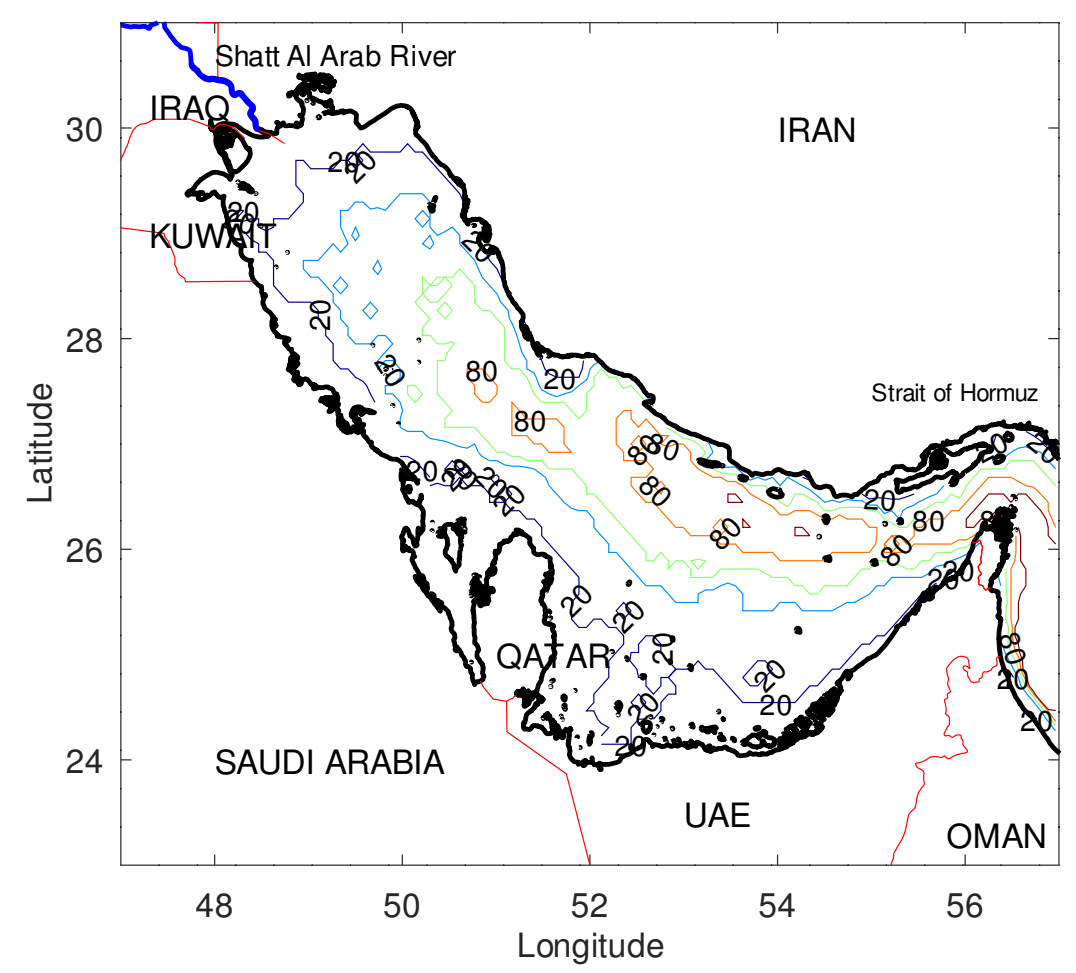

Figure 1. Map of the APG, which corresponds to the present model domain. Isobaths of 20, 40, 60, 80 and $100 \mathrm{~m}$ are drawn.

Table 1. Model availability.

\begin{tabular}{ll}
\hline Program name & APERTRACK \\
Developer & R. Periáñez, University of Sevilla \\
Contact & rperianez@us.es \\
Hardware & Desktop PC \\
Programe code & FORTRAN \\
Cost & Free \\
Availability & http://www.personal.us.es/rperianez \\
\hline
\end{tabular}




\section{Materials and Methods}

The conceptual and mathematical model is described in detail in [5,6], and only some indications are given here. The model is Lagrangian, thus the pollutant release into the sea is simulated by means of a number of particles. Each particle is equivalent to a number of units (for instance $\mathrm{Bq}, \mathrm{kg}$, moles, etc), and trajectories are calculated during the simulated period. The transport model considers physical transport (three dimensional advection due to water currents and three dimensional mixing due to turbulence) plus decay and interactions of pollutants with bed sediments (adsorption/desorption reactions).

Turbulent mixing, decay and exchanges of contaminants between water and sediment are described through a stochastic method whose details can be seen in a number of papers (see for instance [7]). Water currents, which are responsible of advective transport, are due to tides and density driven (baroclinic) circulation. Exchanges of pollutants between water and sediments are described in a dynamic way using kinetic transfer coefficients. These are deduced from the equilibrium distribution coefficient, $k_{d}$ as explained in earlier works (see [9]for instance). The International Atomic Energy Agency (IAEA) [8] provides recommended $k_{d}$ values for a large number of elements.

Advection due to the current in a Lagrangian model is computed solving the following equation for each particle:

$$
\begin{aligned}
& \Delta x=U \Delta t \\
& \Delta y=V \Delta t
\end{aligned}
$$

where $\Delta t$ is time step in the Lagrangian model, $\Delta x$ and $\Delta y$ are the changes in particle position $(x, y) ; U$ and $U$ are water velocity components (east-west and south-north directions respectively) at the particle position and depth and for the corresponding calculation time step, since currents change in time. These currents are the addition of baroclinic currents (downloaded from HYCOM model) and tidal currents and residuals derived from the tidal model. This is explained below.

The maximum size of the horizontal step given by the particle due to turbulent mixing, $D_{h}$, is [7]:

$$
D_{h}=\sqrt{12 K_{h} \Delta t}
$$

in the direction $\theta=2 \pi R A N$, where $K_{h}$ is the horizontal diffusion coefficient (set as 10 $\mathrm{m}^{2} / \mathrm{s}$ ) and $R A N$ is an uniform random number between 0 and 1 . This equation gives the maximum size of the step. The real size at a given time and for a given particle is obtained multiplying the equation by another independent random number. This is required to be sure that a Fickian diffusion process is simulated. Time step used to integrate the Lagrangian model was set as $\Delta t=600 \mathrm{~s}$.

Similarly, the size of the vertical step is [7]:

$$
D_{v}=\sqrt{2 K_{v} \Delta t}
$$

given either upward or downward. $K_{v}$ is the vertical diffusion coefficient, set as $1.0 \times 10^{-5}$ $\mathrm{m}^{2} / \mathrm{s}$.

Decay of the pollutant is solved with a stochastic method [7] in which decay probability is defined as:

$$
p_{d}=1-e^{-\lambda \Delta t}
$$

where $\lambda$ is the pollutant decay constant. A new random number is generated. If $R A N \leq p_{d}$ the particle decays and is removed from the computation. A similar procedure is used to solve uptake/release processes between water and sediments since they are treated as decay processes from water to sediment or from sediment to water. The constants for these processes are the kinetic transfer coefficients mentioned above and details of the method may be seen for instance in $[6,7,9]$.

A two dimensional depth-averaged model was used to simulate tides in the APG. Calculated elevations and currents are treated through standard tidal analysis [11] and 

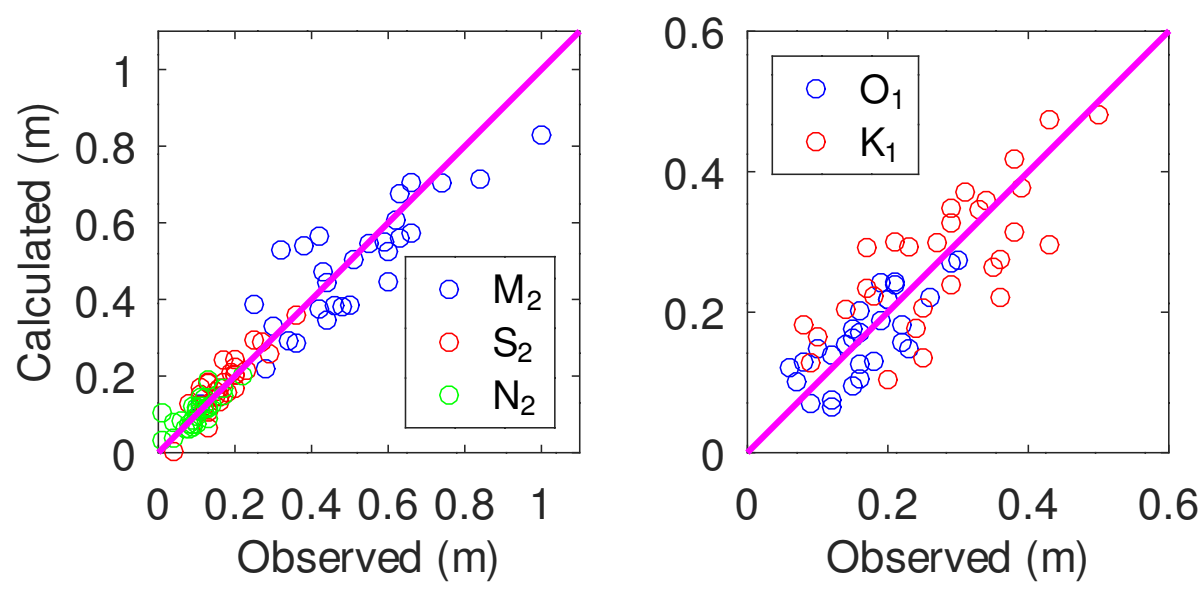

Figure 2. Comparison between calculated and observed amplitudes for the semidiurnal (left) and diurnal (right) constituents considered in the model.

tidal constants (amplitudes and phases) are then calculated and stored for each grid cell in the computational domain. Five constituents were considered: three semidiurnal $\left(M_{2}\right.$, $S_{2}$ and $\left.N_{2}\right)$ and two diurnal $\left(K_{1}\right.$ and $\left.O_{1}\right)$. The Eulerian residual transport is calculated to obtain tidal residual currents [10]. The tidal model is depth-averaged, thus it provides averaged currents over the water column. A standard current profile is used to generate a vertical structure in the tidal current fields, since these currents decrease from sea surface to the bottom because of friction. Details may be seen in [11] for instance. A comparison between observed [12] and calculated elevations for the five constituents may be seen in Figure 2. Agreement is generally good; higher discrepancies appear at some locations, but they are most likely due to the relatively coarse resolution of the model: tides where simulated using the same $0.08^{\circ}$ grid as used in HYCOM model (see below).

Once that amplitudes and phases (adapted phase, i.e., for the local time meridian) for each grid cell and constituent (calculated from the tidal analysis) are known, the nodal factor $f_{i}$ and equilibrium argument $V_{i}$ of each constituent $i$ at Greenwich are used to evaluate the exact tidal state during each time step of the pollutant simulation and location in the APG. The procedure is described in detail in [13]. Nodal factors and equilibrium arguments for year 2021 are used, meaning that the beginning of this year is $t=0$. Of course, values for any other year may be used. The main equation is:

$$
Z(t)=H_{0}+\sum_{i=1}^{5} G_{i} f_{i} \cos \left(w_{i} t-g_{i}+V_{i}\right)
$$

where $H_{0}$ is the location datum, $w_{i}$ is frequency of constituent $i, G_{i}$ and $g_{i}$ are amplitude and phase for the corresponding location (these quantities are obtained from the tidal analysis), $f_{i}$ is nodal factor and $V_{i}$ the equilibrium argument of the constituent at Greenwich. Note that the sum extends to 5 since this is the number of included constituents. This same procedure is applied to tidal currents.

HYCOM (Hybrid Coordinate Ocean Model) [14], model was used to obtain baroclinic circulation in the APG. HYCOM is a primitive equation general circulation model with 40 vertical layers increasing in thickness from the surface to the sea bottom and $0.08^{\circ}$ horizontal resolution in both latitude and longitude. Daily current data was obtained from HYCOM data server. ${ }^{1}$ This model was already used to study circulation in the APG [15].

The domain of the model extends from $23^{\circ} \mathrm{N}$ to $31^{\circ} \mathrm{N}$ in latitude, from $47^{\circ} \mathrm{E}$ to $57^{\circ} \mathrm{E}$ in longitude, and may be seen in Figure 1. The number of Lagrangian particles in the model is 200000 , which provides a good compromise between accuracy and computational speed.

1 https://www.hycom.org/dataserver 
Table 2. Input files which must be modified for each specific simulation. It is required to modify tide-data.dat only if a simulation for other year than 2021 including tidal circulation is to be carried out.

\begin{tabular}{ll}
\hline tide-data.dat & Equilibrium arguments and nodal factors \\
release.dat & Release data and simulation time \\
RN.dat & Contaminant properties (decay constant and $k_{d}$ ) \\
input.dat & Switches to include or not tidal circulation \\
wind.dat & Local wind data \\
\hline
\end{tabular}

Actually, significant differences in results could not be seen if the number of particles was increased. A simulation over three months takes about 10 minutes on a desktop PC working over Ubuntu 18.04 operating system.

A number of files specify the release characteristics (date, time, position in geographic coordinates, depth, magnitude and duration) and simulation time, pollutant properties (decay constant and equilibrium distribution coefficient (which may be obtained from IAEA [8] as mentioned above), and, finally, an optional wind forecast (see next paragraph) and components of the currents to be used: tidal currents and residuals may be individually switched on and off (to allow comparisons if they are included or not in the simulations or to speed them up by removing tides in the calculations). These switches are provided in a specific file named input. dat. A list of the input files which should be modified for a particular simulation is given in Table 2.

In the case of a simulation to assess the effects of an acute release due to an accident, for instance, it may be relevant to include a local wind, which is considered uniform in the release area. Wind data are provided in a file as a number of different "wind episodes" (any number can be used with a maximum of 100), each one characterized by a wind speed, direction and start and end times measured in hours after the pollutant release beginning. This time-evolving wind conditions may be obtained from weather forecasts. It should be commented that HYCOM calculations already include atmospheric forcing. However, the present definition of "wind episodes" gives the opportunity of describing transport in case that an accident occurs, for instance, during a local storm which is not described in HYCOM. The need of adding this local wind in some oil spill simulations in the Red Sea was clearly shown in [5] and was also used in a radionuclide transport model for the same sea [6]. The wind-induced current decreases logarithmically to zero from the surface. The mathematical expression for this profile may be seen for instance in [11]. The use this "local wind" is optional and should be included only if a wind forescast is known and it includes unusual weather conditions. Otherwise atmospheric forcing already included in HYCOM calculations is enough for the transport calculations.

Images of the input files which should be modified for a given simulation can be seen in Figure 3. In these examples, only the $M_{2}$ tidal and residual currents are included (switches at 1 position), but the other constituents are neglected (switches at 0 position). The pollutant is considered stable (decay constant set to zero) and two wind episodes are considered: a $5 \mathrm{~m} / \mathrm{s}$ west wind and a $10 \mathrm{~m} / \mathrm{s}$ blowing from the south.

The model output consists of contaminant concentrations over the model domain in two water layers: a surface layer whose thickness is defined as $10 \mathrm{~m}$, but can be changed by the user in the code, and a deep layer which extends from the bottom of the surface layer to the seabed. Actually, the model provides the pollutant inventory in units $/ \mathrm{m}^{2}$ in the deep layer. Concentrations in bed sediments are provided in a $5 \mathrm{~cm}$ thick sediment layer. In addition, the model provides the position of particles (both in the water column and in sediments) at the end of the simulation. All this information may be drawn with the Octave scripts which are provided with the model. 


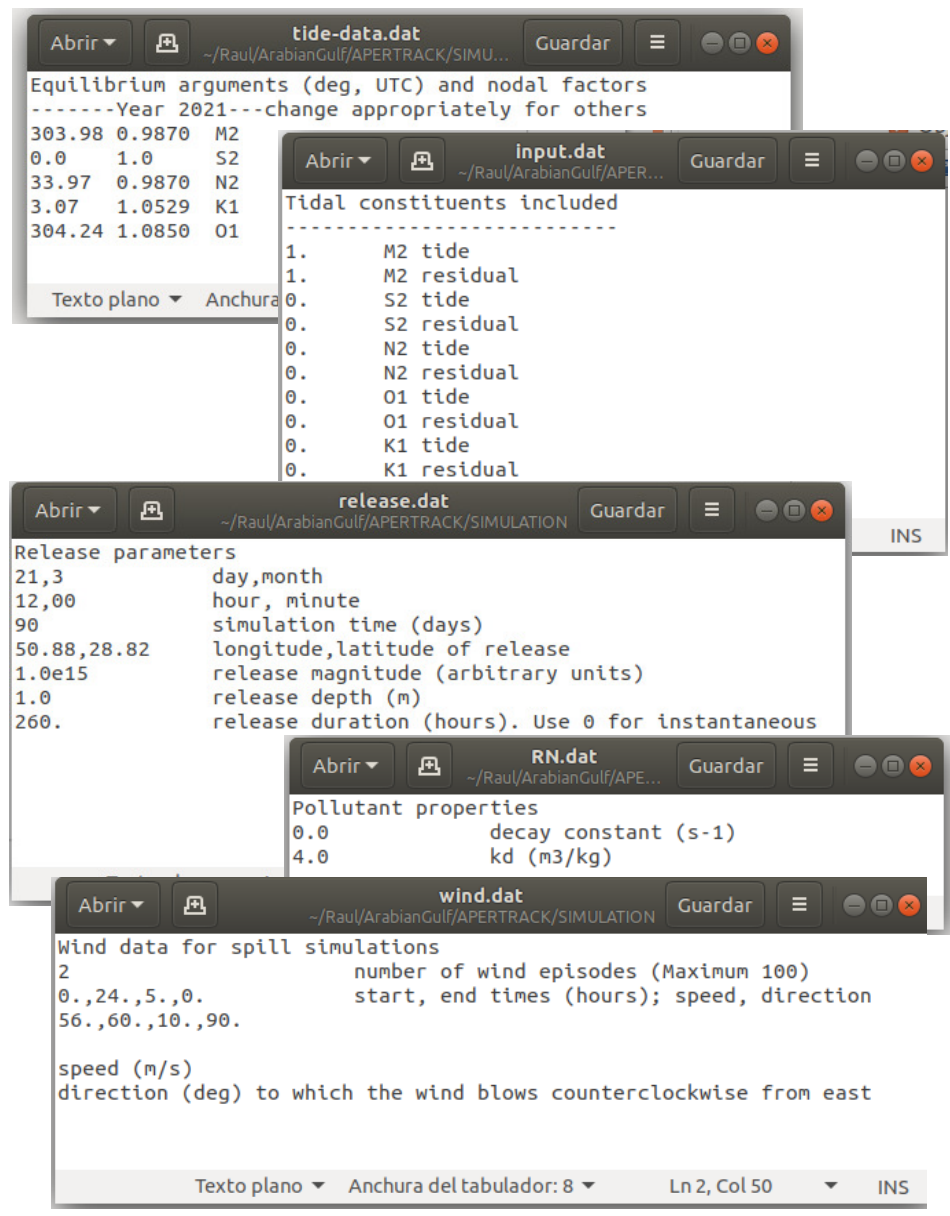

Figure 3. Files which should be edited for a given simulation. 

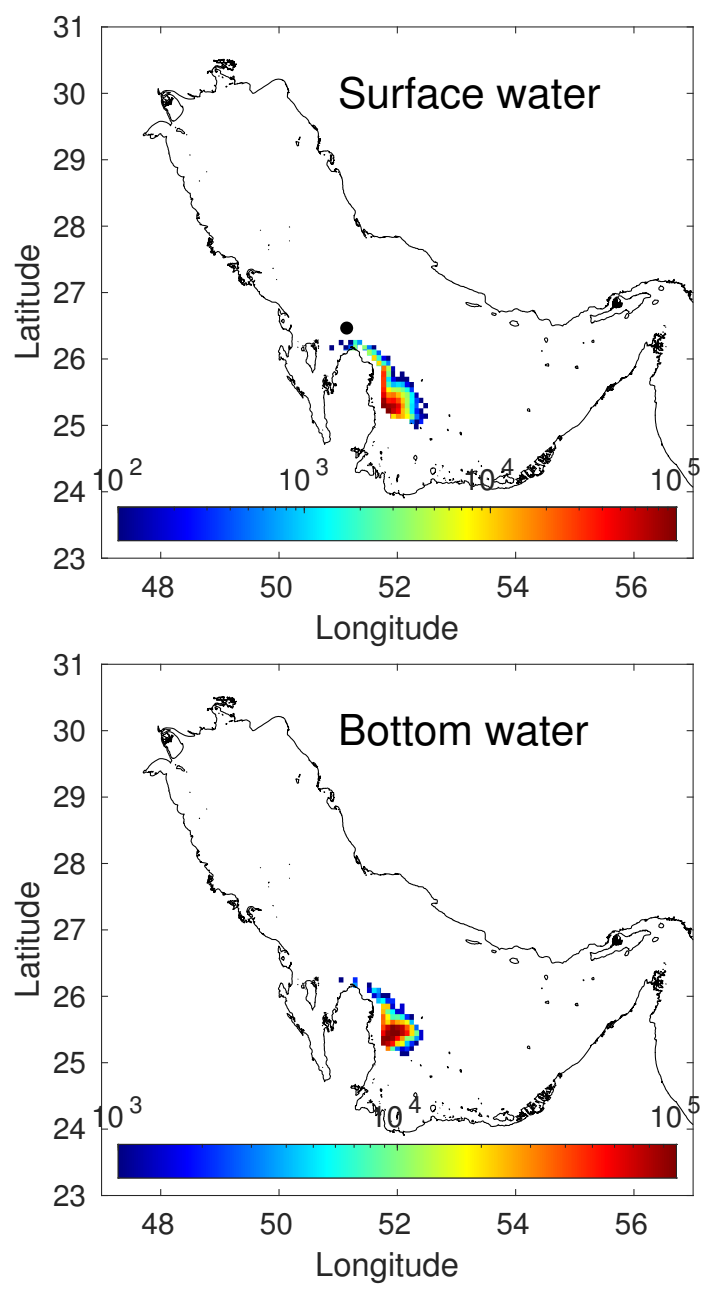

Figure 4. Calculated concentrations in the surface layer (units $/ \mathrm{m}^{3}$ ) and inventory (units $/ \mathrm{m}^{2}$ ) in the bottom water layer for the dissolved pollutant release after 30 days. The black dot in the surface water map indicates the release point.

\section{Results}

Some examples of the model performances are presented now based on hypothetical releases. A release occuring near the coast, due to a ship accident for instance, was supposed to occur at position with coordinates $51^{\circ} \mathrm{E}, 26.5^{\circ} \mathrm{N}$. The accident occurred at 12:00 hours local time in September 15th, 2021. The surface spill lasted 168 hours (one week) and 30 days were simulated. The magnitude of the spill was arbitrarily fixed as $1.0 \times 10^{15}$ units. In the first experiment a stable chemical pollutant (decay constant set to zero) was considered.

In a first example the pollutant remains in dissolved form, without adsorption in sediments (distribution coefficient set to zero). Finally, the 5 tidal constituents with their residuals were considered. A wind forescast was not used in this simulation. Results of this simulation are presented in Figure 4, where maps showing the calculated pollutant distributions in the surface and bottom water layers are shown. The pollutant is transported to the south, reaching a long extension of the coast. The distributions in the bottom water reflects that of the surface.

In a second experiment exactly the same hypothetical accident was simulated, but it was supposed that the released contaminant was $\mathrm{Hg}$, a toxic heavy metal whose distribution coefficient is equal to $30 \mathrm{~m}^{3} / \mathrm{kg}$ [8], thus it presents a high affinity to be fixed to the sediments. Results of this simulation are presented in Figure 5, where a map showing the calculated concentrations in sediments (units $/ \mathrm{kg}$ ) is added. Due to the metal adsorption on the sediment, concentrations in the surface water are lower than in the case of the dissolved 
pollutant (note that the magnitude of the release is the same in both cases, as well as color scales in Figures 4 and 5). In addition, pollutants with high affinity to the sediments are more immobile than those remaining in solution. This can be clearly seen comparing the distributions in the bottom water layer: in the case of $\mathrm{Hg}$ the peak concentrations remain in the area near the spill location.

A final numerical experiment was carried out to illustrate the effects of a local wind due to a local storm, for instance. The $\mathrm{Hg}$ simulation described before was repeated, but it was considered that during the first 5 days a $5 \mathrm{~m} / \mathrm{s}$ was blowing from the west, then increased speed to $20 \mathrm{~m} / \mathrm{s}$ and changed direction to a northwards wind. Results are presented in Figure 6. Obviously, the pollutant is initially transported to the central APG due to the west wind and later there is a significant transport to the north caused by the south wind. Actually some $\mathrm{Hg}$ approaches the eastern coast of the APG and a spot of contaminated sediment is apparent there.

\section{Discussion}

The transport model is easy to setup for any situation since just requires the modification of a few input files specifying the pollutant properties (decay constant and distribution coefficient) and release characteristics (magnitude, location, date, time and duration). Running times are short (a few minutes for a several day long simulation) even on a desktop PC, which makes it appropriate for a rapid assessment of a hypothetical accident occurring in the APG. The model is based upon sound and solid mathematical and numerical approaches which have been widely validated before (see $[6,9,10]$ for instance).

The geochemical behaviour of contaminants should be described within marine transport models, since the resulting concentrations depend on the affinity of the pollutant to be fixed to the sediment. This was clearly shown with the simulations carried out comparing the dissolved contaminant and with $\mathrm{Hg}$.

The present model only provides pollutant concentrations in abiotic compartments (surface and deep waters and sediments). A further step will incorporate a foodweb model which could describe the adsorption of such pollutants by fish. Advances in this topic are presented for instance in [16].

Funding: This research was partially fundeded by the Spanish Ministerio de Ciencia, Innovación y Universidades project PGC2018-094546-B-I00 and Junta de Andalucía (Consejería de Economía y Conocimiento) project US-1263369.

Conflicts of Interest: The authors declare no conflict of interest. The funders had no role in the design of the study.

\section{Abbreviations}

The following abbreviations are used in this manuscript:

APG Arabian/Persian Gulf

IAEA International Atomic Energy Agency

HYCOM Hybrid Coordinate Ocean Model 

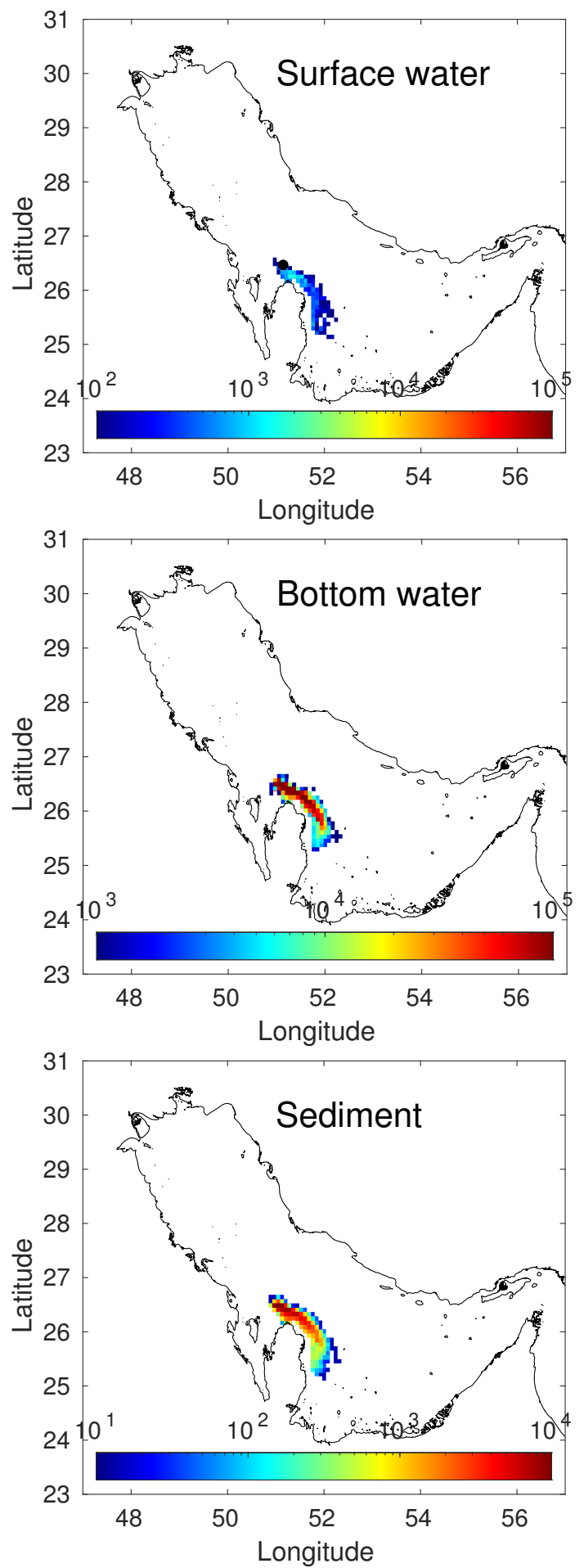

Figure 5. Calculated concentrations in the surface layer (units $/ \mathrm{m}^{3}$ ), inventory (units $/ \mathrm{m}^{2}$ ) in the bottom water layer and concentrations in sediments (units $/ \mathrm{kg}$ ) for the $\mathrm{Hg}$ release after 30 days. The black dot in the surface water map indicates the release point. 

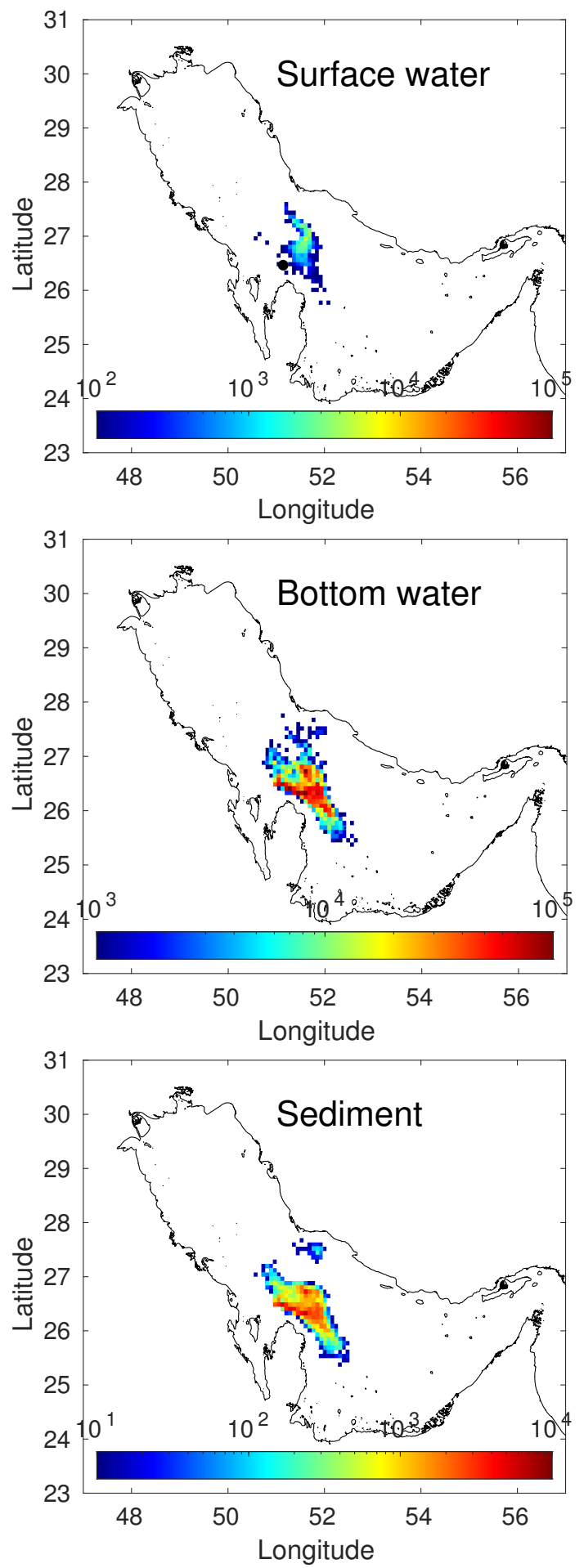

Figure 6. The same as Figure 5 but adding winds due to a local storm. See the main text for details. 


\section{References}

1. Abdi, M.R., Faghihian, H., Kamali, M., Mostajaboddavati, M., Hasanzadeh, A.. Distribution of natural radionuclides on coasts of Bushehr, Persian Gulf, Iran. Iranian Journal of Science E Technology, Transaction A, 2006, 30, 259-269.

2. Al-Ghamdi, H., Al-Muqrin, A., El-Sharkawy, A., 2016. Assessment of natural radioactivity and ${ }^{137} \mathrm{Cs}$ in some coastal areas of the Saudi Arabian gulf. Mar. Pol. Bulletin, 2016, 104, 29-33.

3. Uddin, S., Fowler, S.W., Behbehani, M., Al-Ghadban, A.N., Swarzenski, P.W., Al-Awadhi, N. A review of radioactivity in the Gulf region. Mar. Pol. Bulletin, 2020, 159, 111481.

4. Al-Rabeh, A.H., Lardner, R.W., Gunay, N. Gulfspill Version 2.0: a software package for oil spills in the Arabian Gulf. Environ. Mod. E Soft., 2020, 15, 425-442.

5. Periáñez, R.. A Lagrangian oil spill transport model for the Red Sea. Ocean Eng., 2020, 217, 107953.

6. Periáñez, R. Models for predicting the transport of radionuclides in the Red Sea. J. Environ. Radioact., 2020, 223-224, 106396.

7. Periáñez, R., Elliott, A.J. A particle tracking method for simulating the dispersion of non conservative radionuclides in coastal waters. J. Environ. Radioact., 2002, 58, 13-33.

8. IAEA. Sediment Distribution Coefficients and Concentration Factors for Biota in the Marine Environment. Technical Reports Series 422, 2004, Vienna, Austria.

9. Periáñez, R., Casas-Ruíz, M., Bolívar, J.P. Tidal circulation, sediment and pollutant transport in Cádiz Bay (SW Spain): a modelling study. Ocean Engineer., 2013, 69, 60-69.

10. Periáñez, R., Pascual-Granged, A. Modelling surface radioactive, chemical and oil spills in the Strait of Gibraltar. Comp. and Geosc., 2008, 34, 163-180.

11. Pugh, D.T. Tides, Surges and Mean Sea Level. Wiley, Chichester, UK, 1987, p. 472.

12. Pous, S, Carton, X., Lazure, P. A process study of the tidal circulation in the Persian Gulf. Open Journal of Marine Science, 2012, 2, 131-140.

13. Parker, B.B.. Tidal Analysis and Prediction. NOAA Special Publication, NOS CO-OPS 3, 2007, US.

14. Bleck, R., An oceanic general circulation model framed in hybrid isopycnic-Cartesian coordinates. Ocean Mod., 2001, 4, 55-88.

15. Yao, F., Johns, W.E. A HYCOM modeling study of the Persian Gulf: 1. Model configurations and surface circulation. J. Geophys. Res., 2010, 115, C11017.

16. de With, G., R. Bezhenar, V. Maderich, Y. Yevdin, M. Iosjpe, K.T.Jung, F. Qiao, R. Periáñez. Development of a dynamic food chain model for assessment of the radiological impact from radioactive releases to the aquatic environment. J. Environ. Radioact., 2021, $233,106615$. 